\title{
Stark Effect in Multielectron Systems: Non-Existence of Bound States
}

\author{
I. M. Sigal \\ Department of Mathematics, University of Toronto, Toronto M5S 1A1, Canada
}

\begin{abstract}
We prove non-existence of bound states for a class of $N$-body systems in homogeneous electric fields. This class includes atoms and Born-Oppenheimer molecules. This result in conjunction with a stability result of [HS] implies existence of resonances for such systems.
\end{abstract}

\section{Introduction}

Though the quantum N-body problem was intensively studied for the past 20 years, the field still abounds with many basic problems. For instance, physical intuition suggests that a system placed in an external field which, in a certain direction, pulls it apart overcoming the attraction between particles should not have bound states. In particular, a homogeneous electric field applied to a system containing charged particles of opposite charges should break the bound states of this system. So far this rather obvious statement is proven only for two (one)-particle systems ([T, AH, HS] see also [A]). The purpose of this article is to prove that for a large class of $N$-body systems including atoms and Born-Oppenheimer molecules, there are no bound states in the presence of homogeneous electric fields. This result coupled with the stability result of [HS] (see also [Hu]) implies the existence of resonances in such systems. It is shown in [Sig] that these resonances have exponentially small (in the inverse of the strength of electric field) width. The latter is determined by the width, in an appropriate Agmon metric, of the barrier that the system has to penetrate. This shows that establishing non-vanishing of this width is a delicate matter.

Note the principal difference between the one (two)-body and $N$-body problems. In the one-body case, the total potential acting on the particle in question is $V(x)-E \cdot x$, where $E$ is the electric field strength (times the charge of the particle). Hence the force acting on the particle in the $E$ direction is

\footnotetext{
* Research partially supported by NSERC under Grant No. A7901 and NSF under Grant No. DMS 8507040
} 


$$
-\hat{E} \cdot \nabla V(x)+|E|
$$

where $\hat{E}=E /|E|$. It is positive (close to $|E|$ ) for $|x|$ sufficiently large, provided $|\nabla V(x)|$ vanishes as $|x| \rightarrow \infty$ (in fact, vanishing of $\hat{E} \cdot \nabla V(x)$ in the direction of field would suffice). In other words, if the particle is in the half-space $E \cdot x>0$ and sufficiently far from the origin, it is pulled off to $\infty$ by the electric force. In the $N$-body case, the total potential is $\Sigma V_{i j}\left(x_{i}-x_{j}\right)-\Sigma e_{i} E \cdot x_{i}$, and the total force acting on the $i^{\text {th }}$ particle is the direction of $E$ is

$$
-\sum_{j \neq i} \hat{E} \cdot \nabla V_{i j}\left(x_{i}-x_{j}\right)+e_{i}|E| \text {. }
$$

In general, for not so large $|E|$, this force pulls the particle to $\infty$ only when this particle is away from other particles. Put differently, along the planes

$$
\left\{x \mid x_{i}=x_{j} \text { for some } j\right\}
$$

this force can be negative and push the particle back to the origin.

In our proof we adopt a modification of the method of $\left[\mathrm{FH}(\mathrm{H}-\mathrm{O})^{2}\right]$ and $[\mathrm{FH}]$ (see [RSIV] for a review of related works), where the absence of the positive energy bound states is shown for a large class of systems. The $N$-body systems we consider are specified as follows. $N$ particles have "arbitrary" interactions with arbitrary fixed centres and repulsive (at least, in the direction of the electric field) interactions with each other. In fact, our assumptions are even weaker than that since we incorporate the electric field into the potentials and its effect shows only through a general estimate. Our approach is not intrinsically an $N$-body one. Instead of using the geometry of the $N$-body problem we use that the interactions between moving particles are repulsive. This fact allows us to reduce the problem to one of independent particles interacting only with the fixed centres. Though we cannot separate variables and reduce the problem to the one-particle one, as it is normally done, the resulting problem, given some additional tricks, can be easily managed. The underlying intuition is that the repulsive interaction between moving particles will prevent them from sticking together, i.e. will keep the system away from the planes (1.1) along which the electric force is not effective. Note that repulsive estimates instead of geometric analysis were used in [L] in an analysis of $N$-body scattering.

The paper is organized as follows. The second section deals with the 1-dimensional one-particle case. Here we develop most of our machinery. The third section treats the $n$-dimensional one-particle case. This treatment is, in fact, reduced to a few remarks concerning essentially the notation. No new ideas or tools are introduced here. The fourth section deals with the general case, i.e. the class of $N$-body systems described above. In the fifth section we discuss generalizations to potentials including electric fields vanishing at infinity as $0\left(|x|^{-\alpha}\right)$ with $\alpha<2$.

\section{One-Dimensional One-Particle Problem}

a. Hamiltonian. We define a class of Schrödinger operators (Hamiltonians) on $L^{2}(\mathbf{R})$ which includes one-dimensional Stark Hamiltonians. Let $p=-i(d / d x)$, and 
define

$$
H=p^{2}+V(x) \text { on } \quad L^{2}(\mathbf{R}) .
$$

The potential $V(x)$ will be assumed real and such that $H$ is self-adjoint and the force $-V^{\prime}$ pulls the particle which is on the interval $x>R$ to $\infty$ :

(A) $V=V_{1}+V_{2}$ with $V_{1} \in L_{\text {loc }}^{2}(\mathbf{R}), V_{1}(x) \geqq-c \max (x, 0)$ for some $c>0$ and $V_{2} \in L^{2}(\mathbf{R})+L^{\infty}(\mathbf{R})$.

(B) $\quad V$ has a distributional derivative satisfying: $-V^{\prime}(x)$ is bounded and $\geqq \delta$ for $x \geqq R$ for some $\delta, R>0$.

By the Faris-Lavine theorem (see e.g. [RSII]), $H$ is self-adjoint due to condition (A). This condition will be also used to guarantee the unique continuation property for $H$ (see [ABG, H, SchS] and the references therein). Condition (B) on the force will be used to show that $H$ has no bound states. Note that the usual Stark potential

$$
V(x)=W(x)-E x
$$

where $W(x)$ is a one-body potential with $W^{\prime}(x)$ vanishing as $x \rightarrow \infty$, obeys this condition:

$$
-V^{\prime}(x)=E-W^{\prime}(x) \rightarrow E>0 \quad \text { as } \quad x \rightarrow \infty .
$$

We present some properties of the domain of $H$ used henceforth. We have

$$
D(H) \subset \text { local Sobolev space of order } 2 .
$$

This follows readily from the local $H$-boundedness of $V$ with the relative bound 0 . Next,

$$
D(H) \subset D\left(p^{2}\langle x\rangle^{-1 / 2}\right),
$$

where $\langle x\rangle=\left(1+x_{+}\right)$and $x_{+}=\max (x, 0)$. We prove this embedding in Appendix.

b. Exponential Bounds. In this section we prove exponential bounds on bound states of $H \ldots$ as if the latter exist. These bounds are used in the next section to prove absence of bound states.

Theorem 2.1. Let $\psi$ be a bound state of $H$. Then $e^{\alpha x+} \psi \in L^{2}$ for all $\alpha \geqq 0$.

This result is expected; in a bound state, the particle tries to avoid the region in which force pulls it to $\infty$.

Idea of the Proof. First we defined a smooth version of $x_{+}$shifted to the right

$$
\begin{aligned}
F(x) & =\left\{\begin{array}{lll}
x-R-1 / 2 & \text { if } & x \geqq R+1, \\
0 & \text { if } & x \leqq R
\end{array}\right. \\
0 & \leqq F, \quad 0 \leqq F^{\prime} \leqq 1, \quad F^{\prime \prime} \geqq 0
\end{aligned}
$$

The graph of $F^{\prime}$ is shown in Fig. 1:

Now assume on the contrary that for some $\alpha>0$,

$$
e^{\alpha F} \psi \notin L^{2} .
$$

We absorb this fixed $\alpha$ into $F$ (i.e. we write $F$ for $\alpha F$ ). Let for $t>2 R+2, F_{t}(x)$ be 

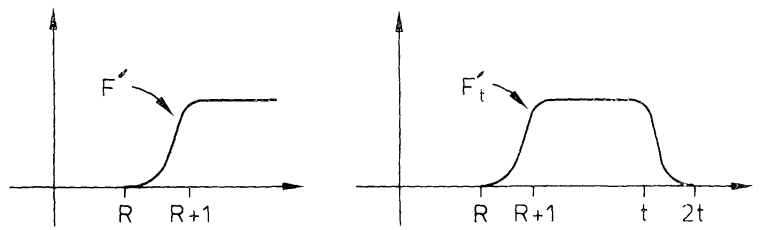

Fig. 1

smooth and obeying

$$
F_{t}(x)=\begin{array}{lll}
F(x) & \text { if } & x \leqq t \\
\alpha \mathrm{t} & \text { if } & x \geqq 2 t,
\end{array}
$$

$F_{t}^{(k)}(x)=O\left(t^{-k+1}\right), k \geqq 1$, and $F_{t}^{\prime \prime}(x) \leqq 0$ for $x \geqq R+1$ and $0 \leqq F_{t}^{\prime} \leqq F^{\prime}$ (the derivatives are taken in $x$ ) (see Fig. 1). Form the wave packets

$$
\psi_{t}=\frac{e^{F_{t}} \psi}{\left\|e^{F_{t}} \psi\right\|} .
$$

Because of (2.3),

$$
\left\|\psi_{t}\right\|=1, \quad \psi_{t} \stackrel{w}{\rightarrow} 0 .
$$

This relation suggests that $\psi_{t}$ is a scattering sequence. Define the self-adjoint operator

$$
A^{F}=\frac{1}{2}\left(F^{\prime} p+p F^{\prime}\right)
$$

This is just the momentum localized to the region where the force $-V^{\prime}$ pulls the particle to $+\infty$ (see Condition (B)). The commutator $i\left[H, A^{F}\right]$ is, up to local terms, the force in this region. As expected, the local terms vanish along the sequence $\psi_{t}$ which allows us to show that

$$
\left\langle i\left[H, A^{F}\right]\right\rangle_{t} \geqq \delta_{1} \quad \text { for } \quad t \text { suffic. large, }
$$

and for some $\delta_{1}>0$. Here and henceforth we use the notation

$$
\langle A\rangle_{t}=\left\langle\psi_{t}, A \psi_{t}\right\rangle \text {. }
$$

On the other hand we show that

$$
\left\langle i\left[H, A^{F}\right]\right\rangle_{t} \leqq C t^{-1} .
$$

The latter estimate is a Virial type result which witnesses the bound-state nature of $\psi_{t}$. It exploits the eigenequation

$$
\left(H^{F_{t}}-E\right) \psi_{t}=0,
$$

where $E$ is the eigenvalue of $H$ corresponding to the eigenfunction $\psi$ and

$$
H^{F} \equiv e^{F} H e^{-F}=H-\left(F^{\prime}\right)^{2}+2 i A^{F} .
$$

Contradicting Eqs. (2.7) and (2.8) show that $e^{F} \psi \in L^{2}$, which implies the statement of the theorem. 
Proof of Theorem 2.1. First we prove Eq. (2.7). We use the equation

$$
i\left[H, A^{F}\right]=-F^{\prime} V^{\prime}+2 p F^{\prime \prime} p-\frac{1}{2} F^{i v},
$$

which is obtained by the straightforward evaluation of the commutator. Since $F^{\prime \prime}$ has a compact support and due to Equation (2.2) and since $\left|V^{\prime}\right|$ is bounded on $\operatorname{supp} F^{\prime}, e^{G} u$ is in the domain of $\left[H, A^{F}\right]$ for any $u \in D(H)$ and any smooth $G$ such that $e^{G} u \in L^{2}$. Since $F^{i v}$ has a compact support we have

Hence

$$
\left|\left\langle F^{i v}\right\rangle_{t}\right| \leqq C \frac{\int \psi^{2}}{\int e^{2 F_{t}} \psi^{2}}
$$

$$
\left\langle F^{i v}\right\rangle_{t} \rightarrow 0 \quad \text { as } \quad t \rightarrow 0 .
$$

Since $F^{\prime \prime} \geqq 0$, we have that

$$
p F^{\prime \prime} p \geqq 0 .
$$

Since $F^{\prime \prime}$ has a compact support, $\psi_{t} \in D\left(\left(F^{\prime \prime}\right)^{1 / 2} p\right)$. Next, by $(B), F^{\prime} V^{\prime}$ is bounded. We show that for $t$ suitably large

$$
-\left\langle F^{\prime} V^{\prime}\right\rangle_{t} \geqq \frac{\delta}{2} .
$$

First, observe that $F$ is chosen in such a way that

$$
-F^{\prime} V^{\prime} \geqq \delta F^{\prime}
$$

Next, since $F^{\prime}$ and $F_{t}$ are supported in $[R, \infty)$, we have

Hence

$$
\left\langle F^{\prime}\right\rangle_{t} \geqq 1-\frac{\int_{-\infty}^{R} e^{2 F_{t}} \psi^{2}}{\int_{-\infty}^{\infty} e^{2 F_{t}} \psi^{2}} \geqq 1-\frac{1}{\left\|e^{F_{t}} \psi\right\|^{2}} .
$$

$$
\left\langle F^{\prime}\right\rangle_{t} \rightarrow 1 \text { as } t \rightarrow \infty .
$$

Thus (2.14) follows. Equations (2.11)-(2.14) yield

$$
\left\langle i\left[H, A^{F}\right]\right\rangle_{t} \geqq \frac{\delta}{2} \text { for large } t .
$$

Next we derive a Virial-type result. Let

$$
\langle A\rangle_{G}=\left\langle\psi_{G}, A \psi_{G}\right\rangle \text { with } \psi_{G}=e^{G} \psi .
$$

Lemma 2.2. Let $G$ and $J$ be smooth with bounded derivatives, let $J^{\prime}$ live in $x \geqq R$ and $J^{\prime \prime}$ have a compact support and let $\psi_{G} \in D\left(A^{J}\right) \cap D\left(A^{G}\right)$. Then

$$
\left\langle i\left[H, A^{J}\right]\right\rangle_{G}=-4 \operatorname{Re}\left\langle A^{J} A^{G}\right\rangle_{G}-2\left\langle J^{\prime} G^{\prime} G^{\prime \prime}\right\rangle_{G} .
$$

Proof. Observe first that since supp $J^{\prime \prime}$ is compact, $\psi_{G}$ is in the domain of $\left[H, A^{J}\right]$. Note the eigenequation

$$
\left(H^{G}-E\right) \psi_{G}=0 .
$$


We use a "Virial theorem" for this equation:

$$
0=\operatorname{Im}\left\langle A^{J} \psi_{G},\left(H^{G}-E\right) \psi_{G}\right\rangle .
$$

Due to the equation

$$
H^{G}=H-\left(G^{\prime}\right)^{2}+2 i A^{G},
$$

we have that $\psi_{G} \in D(H)$. Furthermore, the last two equations yield

$$
0=\operatorname{Im}\left\langle A^{J} \psi_{G},(H-E) \psi_{G}\right\rangle-\operatorname{Im}\left\langle A^{J} \psi_{G},\left(G^{\prime}\right)^{2} \psi_{G}\right\rangle-2 \operatorname{Re}\left\langle A^{J} \psi_{G}, A^{G} \psi_{G}\right\rangle .
$$

Using that

and

$$
-\operatorname{Im}\langle A f, B f\rangle=\frac{1}{2}\langle f, i[B, A] f\rangle
$$

$$
\left.i\left[G^{\prime}\right)^{2}, A^{J}\right]=-J^{\prime}\left(\left(G^{\prime}\right)^{2}\right)^{\prime}
$$

we arrive at (2.17).

For special $J$ and $G$ we derive a more detailed relation. Let

$$
\chi_{t}(r)=\left\{\begin{array}{ll}
F_{t}^{\prime}(r) & \text { if } \quad r \geqq 2 R+1 \\
1 & \text { if } \quad r \leqq 2 R+1
\end{array} .\right.
$$

Then

$$
F_{t}^{\prime}=F^{\prime} \chi_{t}
$$

Note that $J=F_{s}$ and $G=F_{t}$ obey conditions of Lemma 2.2 and, due to Eq. (2.2), $\psi_{t}$ is in the domain of $\chi_{t}^{1 / 2} A^{F}$.

Lemma 2.3. The following identity holds

$$
\left\langle i\left[H, A^{F}\right]\right\rangle_{t}=-4\left\|\chi_{t}^{1 / 2} A^{F} \psi_{t}\right\|^{2}-\left\langle 2 F^{\prime 2} F^{\prime \prime}-2 \chi_{t}^{\prime \prime}+\chi_{t}^{2 \prime}\right\rangle_{t} .
$$

Proof. Using (2.18) and computing a simple commutator, we obtain

$$
A^{F_{t}}=\chi_{t} A^{F}-\frac{i}{2} \chi_{t}^{\prime}
$$

Pick $s>3 t$. Taking into account that

$$
F_{s}=F \text { on } \operatorname{supp} F_{t}^{\prime} \text { and } F^{\prime}=1 \text { on } \operatorname{supp} \chi_{t}^{\prime},
$$

we derive

$$
\operatorname{Re}\left(A^{F_{\mathrm{s}}} A^{F_{t}}\right)=A^{F} \chi_{t} A^{F}-\frac{1}{2} \chi_{t}^{\prime \prime} .
$$

Taking $J=F_{s}$ and $G=F_{t}$ in (2.17), using the last expression and taking into account support properties of the functions involved, we arrive at

$$
\left\langle i\left[H, A^{F_{s}}\right]\right\rangle_{t}=\text { r.h.s. of (2.19). }
$$

This together with Lemma 2.4 below completes the proof of Lemma 2.3.

Lemma 2.4. Let $u \in D(H)$. Denote $\langle B\rangle_{u}=\langle u, B u\rangle$. Then

$$
\left\langle\left[H, A^{F_{s}}\right]\right\rangle_{u} \rightarrow\left\langle\left[H, A^{F}\right]\right\rangle_{u} .
$$


Proof. Since due to condition (B), $V^{\prime}$ is bounded on supp $F_{s}^{\prime}$ and on $\operatorname{supp} F^{\prime}$, and since $0 \leqq F_{s}^{\prime} \leqq F^{\prime}$ and $F_{s}^{\prime} \rightarrow F^{\prime}$ pointwise, we have

$$
\left\langle F_{s}^{\prime} V^{\prime}\right\rangle_{u} \rightarrow\left\langle F^{\prime} V^{\prime}\right\rangle_{u}
$$

as $s \rightarrow \infty$. Moreover

$$
\left\langle F_{s}^{(i v)}\right\rangle_{u} \rightarrow\left\langle F^{(i v)}\right\rangle_{u}
$$

Next, we claim that

$$
\left\langle p F_{s}^{\prime \prime} p\right\rangle_{u} \rightarrow\left\langle p F^{\prime \prime} p\right\rangle_{u} .
$$

To prove this we observe first that

$$
F_{s}^{\prime \prime}=F^{\prime \prime}+\chi_{s}^{\prime} .
$$

Note that $\chi_{s}^{\prime} \leqq 0, \chi_{s}^{\prime}=O\left(s^{-1}\right)$ and is supported in $[s, 2 s]$. Define $\phi_{s}=\left(-s \psi_{s}^{\prime}\right)^{1 / 2}$. Then $\phi_{s}=O(1)$ and is supported in $[s, 2 s]$. Taking this into account, we obtain

$$
\left\|\left|\chi_{s}^{\prime}\right|^{1 / 2} p u\right\| \leqq \text { const }\left\|\phi_{s}\langle x\rangle^{-1 / 2} p u\right\| \text {. }
$$

This together with Eq. (2.2) implies that

$$
\left\|\left|\chi_{s}^{\prime}\right|^{1 / 2} p u\right\| \rightarrow 0 \quad \text { as } s \rightarrow \infty
$$

which, by virtue of (2.25), yields (2.24). Equations (2.22)-(2.24) together with (2.11) yield (2.21).

Equation (2.19) implies

$$
\left\langle i\left[H, A^{F}\right]_{t} \leqq-4\left\|\chi_{t}^{1 / 2} A^{F} \psi_{t}\right\|^{2}-2\left\langle F^{\prime \prime} F^{2}\right\rangle_{t}+C t^{-1},\right.
$$

with the constant $C$ independent of $t$. Since $F^{\prime \prime} \geqq 0$ we obtain furthermore

$$
\left\langle i\left[H, A^{F}\right]\right\rangle_{t} \leqq-4\left\|\chi_{t}^{1 / 2} A^{F} \psi_{t}\right\|^{2}+C t^{-1} \leqq C t^{-1} .
$$

This contradicts Eq. (2.16). The contradiction proves Theorem 2.1.

c. Absence of Bound States. In this section we use Theorem 2.1 to prove that, given conditions (A) and (B), $H$ has no bound states. The strategy of the proof is similar to that of the proof of Theorem 2.1. Instead of $t$ we use $\alpha$ as a parameter.

Theorem 2.5. H has no bound states.

Proof. Let $\psi$ be an eigenfunction of $H$ with an eigenvalue $E$. We show that this assumption leads to a contradiction. By Theorem 2.1,

$$
e^{\alpha F} \psi \in L^{2} \text { for all } \alpha \geqq 0 .
$$

Define the wave packets

$$
\psi_{\alpha}=\frac{e^{\alpha F} \psi}{\left\|e^{\alpha F} \psi\right\|} .
$$

We claim that $\psi_{\alpha} \in D(p)$ and therefore $\psi_{\alpha} \in D\left(A^{F}\right)$ (cf. Proposition A.2 of Appendix). Indeed, let $\chi \in C_{0}^{x}$ obey $0 \leqq \chi \leqq 1$ and $\left|\chi^{(n)}\right| \leqq C_{n}$. Set $\phi_{\gamma}=\chi \psi_{\gamma}$. Then $\phi_{\alpha} \in D\left(p^{2}\right)$, 
$\left\langle p^{2} \phi_{\alpha}, \phi_{\alpha}\right\rangle=\left\|p \phi_{\alpha}\right\|^{2}$ and

$$
\left\|p \phi_{\alpha}\right\|^{2}=\left\langle H \phi_{\alpha}, \phi_{\alpha}\right\rangle-\left\langle V \phi_{\alpha}, \phi_{\alpha}\right\rangle .
$$

Since $e^{\beta F} \psi_{\alpha} \in L^{2}$ for all $\beta$ we have, due to condition (A), that

$$
-\left\langle V \phi_{\alpha}, \phi_{\alpha}\right\rangle \leqq C(\alpha)+\left\langle H \phi_{\alpha}, \phi_{\alpha}\right\rangle,
$$

Furthermore, we compute

$$
\left\langle H \phi_{\alpha}, \phi_{\alpha}\right\rangle=E\left\|\phi_{\alpha}\right\|^{2}+\operatorname{Re}\left\langle\chi e^{\alpha F}\left[p^{2}, \chi e^{\alpha F}\right] \psi, \psi\right\rangle=E\left\|\phi_{\alpha}\right\|^{2}+\left\|\left(\chi e^{\alpha F}\right)^{\prime} \psi\right\|^{2} .
$$

Therefore (remember, $\left\|\psi_{\alpha}\right\|=1$ )

$$
\left|\left\langle H \phi_{\alpha}, \phi_{\alpha}\right\rangle\right| \leqq C\left(\alpha^{2}+1\right),
$$

and consequently

$$
\left\|p \phi_{\alpha}\right\|^{2} \leqq C_{1}(\alpha)
$$

which yields $\left\|\chi p \psi_{\alpha}\right\| \leqq C_{2}(\alpha)$ and which, in turn, by the monotone convergence theorem, implies the desired result. The latter and equation

$$
H \psi_{\alpha}=\left(-i \alpha F^{\prime} p+E-2 F^{\prime \prime}+\alpha^{2}\left(F^{\prime}\right)^{2}\right) \psi_{\alpha}
$$

imply $\psi_{t} \in D(H)$.

Denote

$$
\langle A\rangle_{\alpha}=\left\langle\psi_{\alpha}, A \psi_{\alpha}\right\rangle \text {. }
$$

Equation (2.17) with $J=F$ and $G=\alpha F$ yields

$$
\left\langle i\left[H, A^{F}\right]\right\rangle_{\alpha}=-4 \alpha\left\|A^{F} \psi_{\alpha}\right\|^{2}-2 \alpha^{2}\left\langle F^{\prime 2} F^{\prime \prime}\right\rangle_{\alpha} .
$$

Since $F^{\prime \prime} \geqq 0$, this yields

$$
\left\langle i\left[H, A^{F}\right]\right\rangle_{\alpha} \leqq 0 .
$$

On the other hand, Eqs. (2.11), (2.13) and (2.15) give

$$
\left\langle i\left[H, A^{F}\right]\right\rangle_{\alpha} \geqq \delta\left\langle F^{\prime}\right\rangle_{\alpha}-\frac{1}{2}\left\langle F^{i v}\right\rangle_{\alpha} .
$$

Recall, $F^{\prime} \geqq 0$. We show that for some $\delta>0$,

$$
\left\langle F^{\prime}\right\rangle_{\alpha} \geqq \delta_{1} \quad \text { for } \quad \alpha \text { suffic. large. }
$$

We have

$$
\left\langle F^{\prime}\right\rangle_{x}=\frac{\int_{R}^{\infty} F^{\prime} e^{2 \alpha F} \psi^{2}}{\int_{-\infty}^{\infty} e^{2 \alpha F} \psi^{2}} \geqq F^{\prime}(R+1) \frac{\int_{R+1}^{\infty} e^{2 \alpha F} \psi^{2}}{\int_{-\infty}^{\infty} e^{2 \alpha F} \psi^{2}}=F^{\prime}(R+1)(1-l)
$$

where

$$
I=\frac{\int_{-\infty}^{R+1} e^{2 \alpha F} \psi^{2}}{\int_{-\infty}^{\infty} e^{2 \alpha F} \psi^{2}}
$$


We estimate

$$
I \leqq \frac{\int_{-\infty}^{R+1} e^{2 \alpha F} \psi^{2}}{\int_{R+2}^{\infty} e^{2 \alpha F} \psi^{2}} \leqq \frac{e^{2 \alpha F(R+1)}}{e^{2 \alpha F(R+2)}} \frac{\int_{-\infty}^{\infty} \psi^{2}}{\int_{R+2}^{\infty} \psi^{2}}
$$

The first factor on the right-hand side vanishes as $\alpha \rightarrow \infty$ while the second factor is independent of $\alpha$ and finite, provided $\psi \neq 0$ for $x \geqq R+2$. By the unique continuation theorem (see e.g. [ABG, H, SchS]), the latter is the case for $\psi \neq 0$. Thus $I \rightarrow 0$ for $\alpha \rightarrow \infty$, and therefore

$$
\left\langle F^{\prime}\right\rangle_{\alpha} \geqq \frac{1}{2} F^{\prime}(R+1) \equiv \delta_{1}
$$

for $\alpha$ sufficiently large.

Next, since $F^{i v}$ is supported in $(-\infty, R+1]$

$$
\left|\left\langle F^{i v}\right\rangle_{\alpha}\right| \leqq C \frac{\int_{-\infty}^{R+1} e^{2 \alpha F} \psi^{2}}{\int_{-\infty}^{\infty} e^{2 \alpha F} \psi^{2}} .
$$

By the same argument as above (see (2.34)),

$$
\left\langle F^{i v}\right\rangle_{\alpha} \rightarrow 0 \text { as } \alpha \rightarrow \infty .
$$

Equations (2.30), (2.31) and (2.36) imply that

$$
\left\langle i\left[H, A^{F}\right]\right\rangle_{\alpha} \geqq \beta \quad \text { for } \quad \alpha \text { sufficiently large }
$$

for some $\beta>0$ independent of $\alpha$. The last equation contradicts (2.29). This proves Theorem 2.5 .

Remark 2.6. The result of Theorem 2.1 alone without condition (B) on the force suffices to prove Theorem 2.5. However, if we do not use condition (B), we need an extra estimate, e.g.

$$
\left\|A^{F} \psi_{\alpha}\right\| \rightarrow \infty \quad \text { as } \quad \alpha \rightarrow \infty
$$

\section{One Particle in $n$ Dimensions}

Now $p=-i \nabla$ and $p^{2} \equiv|p|^{2}=-\Delta$ (negative Laplacian) on $\mathbf{R}^{n}$. The Schrödinger operator under consideration is

$$
H=p^{2}+V(x) \text { on } L^{2}\left(\mathbf{R}^{n}\right)
$$

where the potential $V(x)$ is a real function on $L^{2}\left(\mathbf{R}^{n}\right)$ obeying the following conditions:

(A) $V=V_{1}+V_{2}$ with $V_{1} \in L_{\text {loc }}^{2}\left(\mathbf{R}^{n}\right)$ and $V_{1}(x) \geqq-a(h \cdot x)_{+}$for some $a>0$ and $h \in \mathbf{R}^{n}$, $V_{2} \in L^{p}\left(\mathbf{R}^{n}\right)+L^{\infty}\left(\mathbf{R}^{n}\right), p>n / 2$ for $n \geqq 4$ and $p=2$ for $n \leqq 3$.

(B) $V \in L_{\text {loc }}^{p}\left(\mathbf{R}^{n}\right), p>n / 2$. 
(C) $V$ has distributional first derivatives satisfying $-h \cdot \nabla V(x)$ is bounded and $\geqq \delta$ for $h \cdot x \geqq R$ for some $\delta, R>0$ and $h$, the same as in (A).

Again, by the Faris Lavine theorem, $H$ is self-adjoint due to condition (A). Condition $(\mathrm{B})$ is needed to guarantee that $H$ has the unique continuation property (see $[\mathrm{ABG}, \mathrm{H}, \mathrm{JK}, \mathrm{SchS}]$ ). Condition $(\mathrm{C})$ is the condition on the force. It is satisfied if $V$ combines the one-body potential $W(x)$ with $\nabla W(x)$ vanishing as $|x| \rightarrow \infty$ and the electric potential $-E \cdot x$ with $E \cdot h>0$.

Applying an orthogonal change of variables (the rotation taking $e^{1}=(1,0, \ldots, 0)$ into $h$ ) we reduce the second part of condition (C) to

$$
-\frac{\partial V}{\partial x^{1}}(x) \text { is bounded and } \geqq \delta \quad \text { for } \quad x^{1} \geqq R,
$$

where $x^{1}$ is the first component of $x$.

Theorem 3.1. Let $\psi$ be a bound state of $H$. Then $e^{\alpha\left(x^{1}\right)+} \psi \in L^{2}\left(\mathbf{R}^{n}\right)$ for all $\alpha \geqq 0$.

The proof of this theorem is exactly the same as the proof of Theorem 2.1 with all action taking place only in the $x^{1}$-direction. For instance,

$$
A^{F}=\frac{1}{2}\left(F^{\prime}\left(x^{1}\right) p^{1}+p^{1} F^{\prime}\left(x^{1}\right)\right)
$$

with $p^{1}=-i\left(\partial / \partial x^{1}\right)$. Using this theorem we show exactly as in Sect. 2.

Theorem 3.2. H has no bound states.

Remark 3.3. A slightly simpler version of the proof is obtained if one takes $p^{1}$ instead of $A^{F}$, one uses then that $\langle f\rangle_{t} \rightarrow 0$ and $\langle f\rangle_{\alpha} \rightarrow 0$ for any $f \in C_{0}^{\infty}$ in order to prove positivity estimates of the type (2.16) and (2.37). This version does not extend, however, to the $N$-body case.

\section{Many-Body Systems}

a. Hamiltonian. We consider a system of $N$ particles (for the sake of notations of the masses $\frac{1}{2}$ ) interacting with a number of fixed centres. The Hamiltonian of such a system is

$$
H=\sum_{1}^{N}\left(p_{l}^{2}+V_{l}\right)+\sum_{i<j} V_{i j} \text { on } L^{2}\left(\mathbf{R}^{n N}\right),
$$

where $p_{j}=-i \nabla_{j}$ with $\nabla_{j}$, the gradient in the variable $x_{j}, V_{i}=V_{i}\left(x_{i}\right)$ and $V_{i j}=$ $V_{i j}\left(x_{i}-x_{j}\right)$, the real potentials. $V_{\imath}$ are assumed to obey conditions (A)- (C) of Sect. 3 with the same $h . V_{i}$ is the potential of interaction of the $i^{\text {th }}$ particle with fixed centers and an external field. The potentials $V_{i j}$ are supposed to obey

$$
\begin{gathered}
V_{i j} \text { and }(h \cdot y) \nabla V_{i j} \in L^{p}\left(\mathbf{R}^{n}\right)+L^{\infty}\left(\mathbf{R}^{n}\right) \\
\text { with } p>\frac{n}{2} \text { for } n \geqq 4 \text { and } p=2 \text { for } n \leqq 3
\end{gathered}
$$

and to be repulsive in the $h$ direction:

$$
(h \cdot y) h \cdot \nabla V_{i j}(y) \leqq 0 .
$$


In fact, we need this condition only for $|h \cdot y| \leqq R_{1}$ for sufficiently large $R_{1}$.

Note that atoms with infinitely heavy nuclei and the Born-Oppenheimer molecules in an electric field $E$ obey the above conditions. In this case

$$
V_{i}(x)=\sum_{j} \frac{-Z_{j} e}{\left|x-R_{j}\right|}-e E \cdot x
$$

for $R_{j} \in \mathbf{R}^{3}$ (positions of the nuclei) and

$$
V_{i j}(x)=\frac{e^{2}}{|x|} .
$$

Again, in order to simplify the notation we apply the same orthogonal transformation (rotation $g$ in $\mathbf{R}^{n}$ taking $e^{1}$ to $\left.h\right)$ to all $x_{i}:\left(x_{1}, \ldots, x_{N}\right) \rightarrow\left(g\left(x_{1}\right), \ldots, g\left(x_{N}\right)\right)$. Then condition (C) on $V_{i}$ reads in this case as

$$
-\frac{\partial V_{i}(y)}{\partial y^{1}} \text { is bounded and } \geqq \delta \quad \text { for } \quad y^{1} \geqq R,
$$

and for some $\delta, R>0$ and condition (4.3) on $V_{i j}$ becomes

$$
y^{1} \frac{\partial V_{i j}}{\partial y^{1}}(y) \leqq 0 .
$$

As in the one body case we have

$$
D(H) \subset \text { loc. Sobolev space of order } 2 .
$$

b. Exponential Bounds. As in the one-body case we want to show that the bound states of $H$, if they exist, decay exponentially in the direction of the field. We begin with introducing a function $F(x)$ measuring this decay similarly to the one-body case. The first impulse to define it as $F(x)=\sum_{i=1}^{N} F_{1}\left(x_{i}^{1}\right)$, where $F_{1}$ is equal $F$ of Sect. 2, must be suppressed. It meets technical difficulties. We define $F(x)$ as follows. Let

$$
g_{i}=\hat{g}\left(x_{i}^{1}\right) \text { and } g=\left(g_{1}, \ldots, g_{N}\right),
$$

where $\hat{g}(s)$ is smooth non-negative function with the following properties

$$
\hat{g}(s)=\left\{\begin{array}{cll}
s-R-1 & \text { for } & s \geqq R+2 \\
0 & \text { for } & s \leqq R
\end{array}\right.
$$

and

$$
0 \leqq \hat{g}^{\prime}(s) \leqq 1, \quad \hat{g}^{\prime \prime}(s) \geqq 0 .
$$

Next $\|g(x)\|$ will denote the Euclidean norm of the vector $g(x):\|g(x)\|=\left(\Sigma \hat{g}\left(x_{i}^{1}\right)^{2}\right)^{1 / 2}$. Now define a smooth non-negative function $\phi$ with the properties

and

$$
\phi(s)=\left\{\begin{array}{cll}
s-R_{1}-\frac{1}{2} & \text { for } & s \geqq R_{1}+1 \\
0 & \text { for } & s \leqq R_{1}
\end{array}\right.
$$

$$
0 \leqq \phi^{\prime} \leqq 1, \quad \phi^{\prime \prime} \geqq 0 .
$$


We choose $R_{1}$ so that

Define

$$
\frac{R_{1}}{\sqrt{N}} \geqq 2 R+10
$$

$$
F(x)=\phi(\|g(x)\|) .
$$

Theorem 4.1. Let $\psi$ be an eigenfunction of $H$. Then $e^{\alpha F} \psi \in L^{2}$ for all $\alpha$.

Proof. We follow closely the proof of Theorem 2.1. Assume on contrary that $e^{\alpha F} \psi \notin L^{2}$ for some $\alpha \geqq 0$ and show that this assumption leads to a contradiction. In what follows we incorporate $\alpha$ into $F$, i.e. write $F$ for $\alpha F$ and $\phi$ for $\alpha \phi$. Define for $t>2 R_{1}+2$ a smooth, positive function $\phi_{t}(\lambda)$ such that $\phi_{t}^{(k)}=O\left(t^{-k+1}\right), k \geqq 1$, for $\lambda \geqq R_{1}+1,0 \leqq \phi_{t}^{\prime} \leqq \phi^{\prime}$ (the derivatives are taken with respect to $\lambda$ ) and

Introduce

$$
\phi_{t}(\lambda)=\phi(\lambda) \quad \text { if } \quad \lambda \leqq t \quad \text { and } \quad=\alpha t \quad \text { if } \quad \lambda \geqq 2 t .
$$

Then

$$
F_{t}(x)=\phi_{t}(\|g(x)\|) \quad \text { and } \quad \psi_{t}=\frac{e^{F_{t}} \psi}{\left\|e^{F_{t}} \psi\right\|} .
$$

$$
\left\|\psi_{t}\right\|=1 \text { and } \quad \psi_{t} \stackrel{w}{\rightarrow} 0
$$

Henceforth we use the following notation

$$
A^{F}=\frac{1}{2}(\nabla F \cdot p+p \cdot \nabla F) .
$$

Our task is to obtain a contradiction by estimating $\left\langle\left[H, A^{F}\right]\right\rangle_{t}$ in two different ways. First we compute

where

$$
i\left[H, A^{F}\right]=2 p(\operatorname{Hess} F) p-\frac{1}{2} \Delta^{2} F-\nabla F \cdot \nabla V,
$$

$$
\text { Hess } F=\text { Hessian of } F \text {. }
$$

We evaluate Hess $F$. We have (remember that $\|g\| \geqq R_{1}$ on $\operatorname{supp} \phi^{\prime}(\|g(\cdot)\|)$ )

$$
\nabla_{i} F=\left(\frac{g_{i}^{\prime} g_{i}}{\|g\|}\right) \phi^{\prime} e_{i}^{1}
$$

with the obvious notation $e_{i}^{1}$. We compute

$$
\frac{\partial^{2} F}{\partial x_{i}^{1} \partial x_{j}^{1}}=-\frac{g_{i}^{\prime} g_{i} g_{j}^{\prime} g_{j}}{\|g\|^{3}} \phi^{\prime}+\delta_{i j} \frac{g_{i}^{\prime 2}+g_{i}^{\prime \prime} g_{i}}{\|g\|} \phi^{\prime}+\frac{g_{i}^{\prime} g_{i} g_{j}^{\prime} g_{j}}{\|g\|^{2}} \phi^{\prime \prime} .
$$

Taking into account that $\phi^{\prime} \geqq 0$ and denoting $\tilde{g}_{i}=\left(g_{i} /\|g\|\right)\left(\phi^{\prime}\right)^{1 / 2}$, we obtain

$$
\sum_{i, j} \xi_{i} \frac{g_{i}^{\prime} g_{i} \phi^{\prime} g_{j}^{\prime} g_{j}}{\|g\|^{2}} \xi_{j}=\left(\sum_{i} \tilde{g}_{i} g_{i}^{\prime} \xi_{i}\right)^{2} .
$$

Using the Schwarz inequality and the fact $\Sigma \tilde{g}_{i}^{2}=\phi^{\prime}$, we obtain

$$
\Sigma \xi_{l} g_{i}^{\prime} g_{i} \frac{\phi^{\prime}}{\|g\|^{3}} g_{j} g_{j}^{\prime} \xi_{j} \leqq \Sigma \frac{\phi^{\prime}}{\|g\|} g_{i}^{\prime 2} \xi_{i}^{2} .
$$


Hence, since $g_{i}^{\prime \prime} \geqq 0$ and $\phi^{\prime} \geqq 0$,

$$
\text { Hess } F \geqq\left[\frac{g_{i}^{\prime} g_{i}}{\|g\|} \phi^{\prime \prime} \frac{g_{j}^{\prime} g_{j}}{\|g\|}\right] .
$$

Since $\phi^{\prime \prime} \geqq 0$, we have that

$$
\text { Hess } F \geqq 0 \text {. }
$$

First, we prove a virial type result. For $t>2 R_{1}+2$ we introduce the smooth function

$$
\theta_{t}(r)=\left\{\begin{array}{ccc}
\phi_{t}^{\prime}(r) & \text { for } & r \geqq 2 R_{1}+1 \\
1 & \text { for } & r \leqq 2 R_{1}+1
\end{array}\right.
$$

As in Sect. 2, $\phi_{t}^{\prime}=\phi^{\prime} \theta_{t}$. It is easy to compute that

$$
\nabla F_{t}=\chi_{t} \nabla F
$$

where $\chi_{t}(x)=\theta_{t}(\|g(x)\|)$.

Lemma 4.2. $\psi_{t}$ is in the domain of $\left[H, A^{F_{s}}\right]$ for all $s$ including $s=\infty$.

Proof. We will use Eq. (4.9). First observe that since $F_{t}$ is bounded and $\nabla F_{t}=$ $O\left(\|g\|^{-1}\right)$, where $\|g\| \|=\left(\|g\|^{2}+1\right)^{1 / 2}$, we have, due to Proposition A.1 of Appendix, $\psi_{t} \in D(H)$. Next, since

$$
\text { Hess } F=O\left(\||g|\|^{-1}\right),
$$

we conclude due to Proposition A.l that

$$
D(H) \subset D\left((\text { Hess } F)^{1 / 2} p\right) .
$$

Thus $\psi_{t}$ is in the domain of $p$ Hess $F p$. Next, we consider the term $-\nabla F \cdot \nabla V$. Using Eq. (4.10), we obtain

$$
-\nabla F \cdot \nabla V_{i}=-\phi^{\prime} \frac{g_{i} g_{i}^{\prime}}{\|g\|} \frac{\partial V_{i}}{\partial x_{i}^{1}}
$$

Since $g_{i}^{\prime}$ is supported in $x_{i}^{1} \geqq R$, the right-hand side is bounded by condition $\mathrm{C}$ (Sect. 3). Hence $\psi_{t}$ is in the domain of $\Sigma \nabla F \cdot \nabla V_{i}$. Next, we compute using (4.10)

$$
-\nabla F \cdot \nabla V_{i j}=-\phi^{\prime} \frac{g_{i}^{2^{\prime}}-g_{j}^{2^{\prime}}}{2\|g\|} \frac{\partial V_{i j}}{\partial x_{i}^{1}} .
$$

By the assumptions on potentials

$$
\left(g^{2^{\prime}}\left(x_{i}\right)-g^{2^{\prime}}\left(x_{j}\right)\right) \frac{\partial V_{i j}\left(x_{i}-x_{j}\right)}{\partial\left(x_{i}^{1}-x_{j}^{1}\right)}
$$

is infinitesimally form-bounded with respect to $p^{2}$ (see e.g. [RSII, Theorem X.20], see also [CFKS, p. 7]). Hence, due to Eq. (4.20),

$$
\Sigma\left\|\nabla F \cdot \nabla V_{i j} \psi_{t}\right\| \leqq \frac{1}{4}\left\|p^{2}\right\| g\left\|^{-1 / 2} \psi_{t}\right\|^{2}+C\left\|\psi_{t}\right\|^{2},
$$

the right-hand side bounded by Proposition A.1 of Appendix. Thus 


$$
\left\|\nabla F \cdot \nabla V \psi_{t}\right\|<\infty
$$

which together with (4.9) and (4.18) shows that $\psi_{t}$ is in the form domain of $\left[\mathrm{H}, \mathrm{A}^{F}\right]$. Replacing in the derivation above $F$ by $F_{s}$ we conclude that $\psi_{t}$ is in the form-domain of $\left[H, A^{F_{s}}\right]$ as well.

Lemma 4.3. For each $t, \psi_{t}$ is in the domain of $\chi_{t}^{1 / 2} A^{F}$ and

$$
\left\langle i\left[H, A^{F}\right]\right\rangle_{t} \leqq-4\left\|\chi_{t}^{1 / 2} A^{F} \psi_{t}\right\|^{2}+C t^{-1},
$$

which $C$ independent of $t$.

Proof. We follow the proof of Lemma 2.3. Since $\chi_{t}$ is supported in $\|g(x)\| \leqq 2 t$ and due to Proposition A.1 we have that $\psi_{t}$ is in the domain of $\chi_{t}^{1 / 2} A^{F}$. Relation (4.16) leads to

$$
A^{F_{t}}=\chi_{t} A^{F}-\frac{i}{2} \nabla F \cdot \nabla \chi_{t}
$$

Pick $s>3 t$. Taking into account that

$$
\begin{array}{lll}
F_{s}=F & \text { on } & \operatorname{supp}\left(\nabla F_{t}\right), \\
\chi_{s}=1 & \text { on } & \operatorname{supp} \chi_{t},
\end{array}
$$

we obtain a generalization of Eq. (2.19):

where

$$
\left\langle i\left[H, A^{F_{s}}\right]\right\rangle_{t}=-4\left\|\chi_{t}^{1 / 2} A^{F} \psi_{t}\right\|^{2}+\left\langle J_{t}\right\rangle_{t}
$$

Using that

$$
J_{t}=-\chi_{t}^{2} \nabla F \cdot \nabla|\nabla F|^{2}-|\nabla F|^{2} \nabla F \cdot \nabla\left(\chi_{t}^{2}\right)+\nabla F \cdot \nabla\left(\nabla F \cdot \nabla \chi_{t}\right) .
$$

$$
\nabla|\nabla F|^{2}=2(\operatorname{Hess} F)(\nabla F)
$$

in the sense of $\mathbb{R}^{n N}$-vectors, we get

$$
\left.\nabla F \cdot \nabla|\nabla F|^{2}=2<\nabla F,(\text { Hess } F) \nabla F\right\rangle \geqq 0,
$$

due to Eq. (4.14). Since

$$
\left|\nabla^{n}\left(\chi_{t}\right)^{k}\right| \leqq \operatorname{const} t^{-n}
$$

we conclude that

$$
J_{t} \leqq \text { const } t^{-1}
$$

Equations (4.25) and (4.29) yield

$$
\left\langle i\left[H, A^{F_{s}}\right]\right\rangle_{t} \leqq-4\left\|\chi_{t}^{1 / 2} A^{F} \psi_{t}\right\|^{2}+C t^{-1} .
$$

Next we show that $\left\langle\left[H, A^{F_{s}}\right]\right\rangle_{t} \rightarrow\left\langle\left[H, A^{F}\right]\right\rangle_{t}$. Since $\phi_{s} \rightarrow \phi$ pointwise and since

$$
\frac{g_{i} g_{i}^{\prime}}{\|g\|} \frac{\partial V_{i}}{\partial x_{i}^{1}}
$$

are bounded, we have due to (4.19) that

$$
\left\langle\Sigma \nabla F_{s} \cdot \nabla V_{l}\right\rangle_{t} \rightarrow\left\langle\Sigma \nabla F \cdot \nabla V_{i}\right\rangle_{t}
$$


This fact together with (4.9), (4.14) and $\left|\Delta^{2} F_{s}\right| \leqq$ const implies that

$$
\left\langle\nabla F_{s} \cdot \nabla V_{i j}\right\rangle \leqq C(t)
$$

with the right-hand side independent of $s$. Since $\hat{g}^{2}(s)$ is monotonically increasing and since $V_{i j}(y)$ is repulsive in the direction $e^{1}$ (see Eq. (4.5)), we have

$$
\left(\hat{g}^{2^{\prime}}\left(x_{i}^{1}\right)-\hat{g}^{2^{\prime}}\left(x_{j}^{1}\right)\right) \frac{\partial V_{i j}}{\partial\left(x_{i}^{1}-x_{j}^{1}\right)} \leqq 0 .
$$

Hence (remember, $\phi_{s}^{\prime} \geqq 0$ )

$$
-\frac{\phi_{s}^{\prime}}{2\|g\|}\left(g_{i}^{2^{\prime}}-g_{j}^{2^{\prime}}\right) \frac{\partial V_{i j}}{\partial x_{i}^{1}} \geqq 0 .
$$

Since $0 \leqq \phi_{s}^{\prime} \leqq \phi$ and $\phi_{s}^{\prime} \rightarrow \phi^{\prime}$, then due to bound (4.32) and Fatou's lemma and the Lebesgue convergence theorem

$$
\left\langle\nabla F_{s} \cdot \nabla V_{i j}\right\rangle_{t} \rightarrow\left\langle\nabla F \cdot \nabla V_{i j}\right\rangle_{t}<\infty .
$$

Note for a future reference that the argument of the previous paragraph implies that

$$
-\nabla F \cdot \nabla V_{i j} \geqq 0 \text {. }
$$

Hence by virtue of (4.31) and (4.34),

$$
\left\langle\nabla F_{s} \cdot \nabla V\right\rangle_{t} \rightarrow\langle\nabla F \cdot \nabla V\rangle_{t}
$$

a $s \rightarrow \infty$. Finally, clearly

$$
\left\langle\Delta^{2} F_{s}\right\rangle_{t} \rightarrow\left\langle\Delta^{2} F\right\rangle_{t}
$$

Now, as in Lemma 2.4 one can show that

$$
\left\langle p \operatorname{Hess} F_{s} p\right\rangle_{t} \rightarrow\langle p \text { Hess } F p\rangle_{t} .
$$

Equations (3.46)-(4.38) together with Eq. (4.9) yield

$$
\left\langle\left[H, A^{F_{s}}\right]\right\rangle_{t} \rightarrow\left\langle\left[H, A^{F}\right]\right\rangle_{t}
$$

as $s \rightarrow \infty$. Equations (4.30) and (4.39) yield (4.17).

Lemma 4.4. For some fixed $\delta_{2}>0$,

$$
\lim _{\overline{t \rightarrow \infty}}\left\langle i\left[H, A^{F}\right]\right\rangle_{t} \geqq \frac{\delta_{2}}{4 N} .
$$

Proof. We estimate every term on the right-hand side of (4.9) separately. We begin with the last term. We claim

$$
-\frac{\phi^{\prime}}{\|g\|} \Sigma g_{i}^{\prime} g_{i} \frac{\partial V_{i}}{\partial x_{i}^{1}} \geqq \frac{\delta}{\sqrt{N}} \phi^{\prime}
$$

For any $x \in \operatorname{supp} \phi^{\prime}$, let $k=k(x)$ be defined by $g_{k}(x)=\max _{j} g_{j}(x)$. Then for this $x$,

$$
g_{k}(x) \geqq \frac{1}{\sqrt{N}}\|g(x)\| .
$$


Hence

$$
g_{k}(x) \geqq \frac{R_{1}}{\sqrt{N}},
$$

and therefore, since $R_{1}>10 \sqrt{N}$, and by the definition of $g_{k}$,

$$
g_{k}^{\prime}(x)=1 \text {. }
$$

Furthermore, since (for the same $x$ )

$$
x_{k}^{1} \geqq \frac{1}{2} \hat{g}\left(x_{k}^{1}\right) \geqq \frac{R_{1}}{2 \sqrt{N}} \geqq 2 R,
$$

we conclude with help of Eq. (4.4) that

$$
-\frac{\partial V_{k}}{\partial x_{k}^{1}}(x) \geqq \delta
$$

Equations (4.43)-(4.45) and the fact that $g_{i}, g_{i}^{\prime} \geqq 0$ for all $i$ yield (4.41).

Collecting Eqs. (4.35) and (4.41), we obtain

$$
-\nabla F \cdot \nabla V \geqq \frac{\delta}{\sqrt{N}} \phi^{\prime} .
$$

Equations (4.9), (4.14) and (4.46) yield

$$
i\left[H, A^{F}\right] \geqq \frac{\delta}{\sqrt{N}} \phi^{\prime}-\Delta^{2} F .
$$

Now we estimate $\Delta^{2} F$. A straightforward computation (similar to (4.11)) yields

$$
\Delta^{2} F=O\left(\|g\|^{-1}\right) \text {. }
$$

(In fact, (4.11) implies this equation.) We show first that if $F_{1}$ is supported in $\{x|||| g \mid \| \leqq \rho\}$ with some $\rho<\infty$, then

Indeed,

$$
\left\langle F_{1}\right\rangle_{t} \rightarrow 0
$$

$$
\left|\left\langle F_{1}\right\rangle_{t}\right| \leqq \mathrm{Ce}^{2 \phi(\rho)} \frac{\int \psi^{2}}{\int e^{2 F_{t}} \psi^{2}} \rightarrow 0 .
$$

Next, we claim that for any $\gamma>0$

$$
\left\langle\|g\| \|^{-\gamma}\right\rangle_{t} \rightarrow 0 \text { as } t \rightarrow \infty \text {. }
$$

Indeed, for any $\delta \rightarrow 0$,

$$
\left\langle\|g\|^{-\gamma}\right\rangle_{t} \leqq \delta^{\gamma}+\left\langle\frac{F\left(\|g\| \leqq \frac{1}{\delta}\right)}{\|g\|^{\gamma}}\right\rangle_{t} .
$$

As before the last term vanishes as $t \rightarrow \infty$, so (4.50) follows. Equations (4.48)-(4.50) 
imply

$$
\left\langle\Delta^{2} F\right\rangle_{t} \rightarrow 0 \quad \text { as } \quad t \rightarrow \infty .
$$

Now Eqs. (4.47) and (4.51) imply

$$
\left\langle i\left[H, A^{F}\right]\right\rangle_{t} \geqq \frac{\delta}{\sqrt{N}}\left\langle\phi^{\prime}\right\rangle_{t}+R_{t},
$$

where $R_{t} \rightarrow 0$ as $t \rightarrow \infty$.

Finally, we estimate $\left\langle\phi^{\prime}\right\rangle_{t}$. Since $\phi^{\prime} \geqq 0$, we have

$$
\left\langle\phi^{\prime}\right\rangle_{t} \geqq \phi^{\prime}\left(R_{1}+1\right) \frac{\int_{|g| \geqq R_{1}+1} e^{2 F_{t}} \psi^{2}}{\int e^{2 F_{t}} \psi^{2}}=\phi^{\prime}\left(R_{1}+1\right)\left(\frac{\int_{\mid g ! \geqq R_{1}+1} e^{2 F_{t}} \psi^{2}}{\int e^{2 F_{t}} \psi^{2}}\right) .
$$

Next

$$
\frac{\int_{|g| \leqq R_{1}+1} e^{2 F_{t}} \psi^{2}}{\int e^{2 F_{t}} \psi^{2}} \leqq e^{2 \phi\left(R_{1}+1\right)} \frac{\int \psi^{2}}{\int e^{2 F_{t}} \psi^{2}} \rightarrow 0 .
$$

The last two relations yield

$$
\left\langle\phi^{\prime}\right\rangle \geqq \delta_{1} \quad \text { for } t \text { sufficiently large }
$$

with $\delta_{1}>0$ independent of $t$. Equations (4.52) and (4.53) yield (4.40).

Equation (4.21) contradicts Eq. (4.40). This proves Theorem 4.1.

Now we proceed to the main result of this paper:

Theorem 4.5. The many-body Hamiltonian $H$ has no bound states.

Proof. We follow the proof of Theorem 2.5. We assume $H$ has a bound state $\psi$ and we show that this assumption leads to a contradiction. Theorem 4.1 implies that

$$
e^{\alpha F} \psi \in L^{2}\left(\mathbf{R}^{n N}\right) \text { for all } \alpha \geqq 0,
$$

with $F$ defined in (4.6). Define

$$
\psi_{\alpha}=\frac{e^{\alpha F} \psi}{\left\|e^{\alpha F} \psi\right\|} .
$$

Since $e^{\beta F} \psi_{\alpha} \in L^{2}$ for $\beta>0$, we have $\psi_{\alpha} \in D(p)^{2} \cap D(H)$ by Proposition A.1. Therefore following the argument given in the proof of Lemma 4.2 we conclude that $\psi_{\alpha}$ is in the domain of $\left[H, A^{F}\right]$.

First, we estimate $\left\langle i\left[H, A^{F}\right]\right\rangle_{\alpha}$ from below. This estimate is based on Eq. (4.47). We begin with the term $\langle\Delta F\rangle_{x}$. We use again Eq. (4.48). We first show that if $F_{1}$ is supported in $\{x \mid\|g\| \leqq \rho\}$, then

$$
\left\langle F_{1}\right\rangle_{\alpha} \rightarrow 0 \text { as } \quad \alpha \rightarrow \infty \text {. }
$$

This relation is slightly more subtle than Eq. (4.49). We have 


$$
\left|\left\langle F_{1}\right\rangle_{\alpha}\right| \leqq C \frac{\int_{g \mid \leqq \rho} e^{2 \alpha F} \psi^{2}}{\int_{g ! \geqq \rho+1} e^{2 \alpha F} \psi^{2}} \leqq C \frac{e^{2 \alpha \phi(\rho)}}{e^{2 \alpha \phi(\rho+1)}} \frac{\int_{|g| \leqq p} \psi^{2}}{\int_{\mid g ! \geqq \rho+1} \psi^{2}} .
$$

Since $\phi(\rho+1)>\phi(\rho)$, the second factor on the right-hand side vanishes as $\alpha \rightarrow \infty$, while the third factor is independent of $\alpha$ and is finite, provided $\psi \neq 0$ on $\{x \mid\|g\| \geqq \rho+1\}$. The latter is guaranteed by the unique continuation theorem (see $[\mathrm{ABG}, \mathrm{H}, \mathrm{JK}, \mathrm{SchS}]$ ). Furthermore

$$
\left\langle\|g\|^{-\gamma}\right\rangle_{\alpha} \rightarrow 0 \quad \text { as } \quad \alpha \rightarrow \infty
$$

for any $\gamma>0$, which is proven exactly in the same way as (4.50). Equations (4.48), (4.54) and (4.55) yield

$$
\left\langle\Delta^{2} F\right\rangle_{\alpha} \rightarrow 0 \quad \text { as } \quad \alpha \rightarrow \infty .
$$

Next we want to show that

$$
\varliminf_{\alpha \rightarrow \infty}\left\langle\phi^{\prime}\right\rangle_{\alpha} \geqq \delta_{1}
$$

for some $\delta_{1}>0$. As in (4.53) we have

$$
\left\langle\phi^{\prime}\right\rangle_{\alpha} \geqq \phi^{\prime}\left(R_{1}+1\right)\left[1-\frac{\int g_{g} \leqq R_{1}+1}{\int e^{2 \alpha F} \psi^{2}}\right] .
$$

Next

$$
\frac{\int_{|g| \leqq R_{1}+1} e^{2 \alpha F} \psi^{2}}{\int e^{2 \alpha F} \psi^{2}} \leqq \frac{\int_{|g| \leqq R_{1}+1} e^{2 \alpha F} \psi^{2}}{\int_{|g| ⿰ R_{1}+2} e^{2 \alpha F} \psi^{2}} \leqq \frac{e^{2 \alpha \phi\left(R_{1}+1\right)}}{e^{2 \alpha \phi\left(R_{1}+2\right)}} \frac{\int_{|g| \leqq R_{1}+1} \psi^{2}}{\int_{\mid g R_{1}+2} \psi^{2}} .
$$

The last expression vanishes by the same argument as before.

Equations (4.47), (4.56) and (4.57) yield

$$
\varliminf_{\alpha \rightarrow \infty}\left\langle i\left[H, A^{\mathrm{F}}\right]\right\rangle_{\alpha} \geqq \frac{\delta_{2}}{\sqrt{N}}
$$

for some $\delta_{2}>0$.

On the other hand we have a Virial type result. Indeed, recall that $\psi_{\alpha} \in D\left(A^{F}\right)$. Hence a straightforward generalization of Eq. (2.28) gives

$$
\left\langle i\left[H, A^{F}\right]\right\rangle_{\alpha}=-4 \alpha\left\|A^{F} \psi_{\alpha}\right\|^{2}-\alpha^{2}\left\langle\nabla F \cdot \nabla|\nabla F|^{2}\right\rangle_{\alpha} .
$$

The last term of this relation was already computed and estimated: Eq. (4.28). Equations (4.59) and (4.28) yield

$$
\left\langle i\left[H, A^{F}\right]\right\rangle_{\alpha} \leqq-2 \alpha\left\|A^{F} \psi_{\alpha}\right\|^{2} \leqq 0 .
$$

This inequality contradicts (4.58) which shows that the bound state $\psi$ for $H$ is impossible. 


\section{Generalizations}

Open problem: To extend the results and proofs above to the case of electric field whose strength vanishes as $|x| \rightarrow \infty$ as $O\left(|x|^{-\alpha}\right)$ with $\alpha<2$. Instead of condition (C) (Sect. 3) one can consider the condition:

(C) $\quad V$ has distributional first derivatives satisfying $-(h \cdot x)^{\alpha}(h \cdot \nabla V(x))$ is bounded and $\geqq \delta$ for $h \cdot x \geqq R$ and $\alpha<2$ for some $\delta, R>0$ and some $h \in \mathbf{R}^{n}$.

In the case when the repulsive part of $V(x)$ is due to an electric field $E(x)$, this condition allows $E(x)=O\left(|x|^{-\alpha}\right)$ with $\alpha<2$. Note again that after an appropriate change of variables condition $\left(\mathrm{C}^{\prime}\right)$ becomes

$$
-\left(x^{1}\right)^{\alpha} \frac{\partial V}{\partial x^{1}}(x) \text { is bounded and } \geqq \delta \quad \text { for } \quad x^{1} \geqq R \quad \text { and } \quad \alpha<2 \text {. }
$$

A more precise statement of this problem is to show that $H$ as defined in Sect. 4 but with condition $(\mathrm{C})$ replaced by condition $\left(\mathrm{C}^{\prime}\right)$ has no bound states. One way to go about this problem is to analyse the commutator $i\left[H, A_{1}\right]$, where, after the change of variables, $A_{1}=\frac{1}{2}\left(p^{1} G\left(x^{1}\right)+G\left(x^{1}\right) p^{1}\right)$ in the one-particle case and an appropriate generalization of this expression along the lines of Sect. 4 , in the many-particle case. Here $G(s)$ is a smooth function such that $G, G^{\prime} \geqq 0$ and $G(s)=0$ for $s \leqq R$ and $=(s-R)^{\alpha}$ for $s \geqq R+1$. One should proceed along the lines of the proofs of the previous section with some additional calculations involving the Virial-type result. We believe, in fact, that there is no bound states even if condition (C) $\left(\right.$ or $\left.\left(\mathrm{C}^{\prime}\right)\right)$ is replaced by the condition

$$
-h \cdot \nabla V(x) \geqq 0 \quad \text { for } \quad h \cdot x \geqq R
$$

for some $h \in \mathbf{R}^{n}$ and some $R>0$.

\section{Appendix}

In this appendix we establish some elementary properties of the domain of $H$. The latter is a many-body Hamiltonian as defined in Sect. 4a. Special cases of the results below apply to the one-body Hamiltonians of Sects. $2 \mathrm{a}$ and 3 . The conditions on potentials we use here are somewhat more general than in the main text and can be further generalized. We assume

(i) $V_{l}=V_{i}^{(1)}+V_{i}^{(2)}$ with $V_{i}^{(1)}(y) \geqq-a y_{+}^{1}$ for some $a \geqq 0$ and $V_{l}^{(2)} \in L^{p}\left(\mathbf{R}^{n}\right)+L^{\infty}\left(\mathbf{R}^{n}\right)$.

(ii) $V_{i j}$ and $y^{1}\left(\partial V_{i j} / \partial y^{1}\right) \in L^{p}\left(\mathbf{R}^{n}\right)+L^{\infty}\left(\mathbf{R}^{n}\right)$. Here and above $p>(n / 2)$ for $n \geqq 4$ and $p=2$ for $n \leqq 3$.

By the Faris-Lavine theorem (see e.g. [RSII]) the N-body Hamiltonian $H$, with these assumptions, is self-adjoint. Moreover, $V_{i}^{(2)}, V_{i j}$ and $y^{1}\left(\partial V_{i j} / \partial x^{1}\right)$ are infinitesimally $p^{2}$ form-bounded (see e.g. [RSII, Theorem X. 19]). 
Proposition A.1. Assume (i) and (ii). Then

$$
D(H) \subset D\left(p^{2}\|g\| \|^{-(1 / 2)}\right) .
$$

Proof. First we show that $D(H) \subset D\left(p\|g\|^{-(1 / 2)}\right)$. Let $u \in D(H)$ and $f=\|g\| \|^{-(1 / 2)} u$. It suffices to show that $f \in D(p)$. Let $\chi \in C_{0}^{\infty}$ with $0 \leqq \chi \leqq 1$ and $\left|\partial^{\alpha} \chi\right| \leqq C_{\alpha}$. Set $v=\chi f$. We use the equation

$$
\|p v\|^{2}=\langle H\rangle_{v}-\langle V\rangle_{v}
$$

with the notation $\langle B\rangle_{v}=\langle v, B v\rangle$. By condition (i),

$$
-\left\langle V_{i}\right\rangle_{v} \leqq\langle\|g(x)\|\rangle_{v}+\left\langle V_{i}^{(2)}\right\rangle_{v} .
$$

Moreover, as was mentioned in the paragraph following conditions (i) and (ii), $V_{i}^{(2)}$ is infinitesimally form $p^{2}$-bounded. As a result we have

$$
-\sum\left\langle V_{i}\right\rangle_{v} \leqq \frac{1}{4}\|p v\|^{2}+C\|u\|^{2} .
$$

Furthermore, by the infinitesimal form boundedness of $V_{i j}$, due to condition (i), we have

$$
\sum\left|\left\langle V_{i j}\right\rangle_{v}\right| \leqq \frac{1}{4}\|p v\|^{2}+C\|v\|^{2} .
$$

Next, commuting $H$ through $\chi \| g^{-(1 / 2)}$ and using that

$$
\operatorname{Re}\left(\chi\|g\|^{-1 / 2} i\left[p^{2}, \chi\|g\| \|^{-1 / 2}\right]\right)=\left|\nabla\|||\|^{-1 / 2} \chi\right|^{2},
$$

we obtain

$$
\left|\langle H\rangle_{v}\right| \leqq\left\langle\|\| g \|^{-1} H\right\rangle_{u} \mid+C\|f\|^{2}
$$

Collecting the estimates above and using Eq. (A.2), we arrive at

$$
\frac{1}{4}\|p v\|^{2} \leqq \text { const. }
$$

This inequality yields $\|\not p f\| \leqq$ const, which, by the monotone convergence theorem, implies that $\left\|p f^{\prime}\right\| \leqq$ const, i.e. $f \in D(p)$.

Now we prove (A.1). Commuting $H$ through $\|g\| \|^{-1 / 2}$, we obtain

$$
H f=\|g\|^{-1 / 2} H \psi-2\left(\nabla\|g\|^{-1 / 2}\right) \cdot \nabla \psi-\left(\Delta\|g\|^{-1 / 2}\right) \psi .
$$

Hence by the result above $f \in D(H)$. Next, similarly to (A.4) and (A.5), we derive

$$
\|V v\| \leqq \frac{1}{2}\left\|p^{2} v\right\|+C\|v\|
$$

which yields

$$
\|v v\| \leqq\|H v\|+C\|v\| .
$$

Commuting $H$ through $\%$ and taking into account that $f \in D(p) \cap D(H)$, we obtain

$$
\|\chi V f\| \leqq C(\|H f\|+\|p f\|+\|f\|) .
$$

Applying again the monotone convergence theorem, we conclude that $f \in D(V)$. Consequently, since $p^{2}=H-V, f \in D\left(p^{2}\right)$, which implies (A.1). 
Proposition A.2. Let $H$ be as in Proposition A.1. Let $\psi$ be an eigenfunction of $H$. Let $F$ be a smooth function with bounded derivatives and such that $\psi_{F} \in D\left(\|\| g\|\|^{n / 2}\right)$. Then $\psi_{F} \in D(H) \cap D\left(p^{n}\right)$. Here $n=1,2$.

Proof. Recall the definition

$$
H_{F}=e^{F} H e^{-F}=H-|\nabla F|^{2}+2 i A^{F} .
$$

Clearly $\psi_{F} \in D\left(H_{F}\right)$ as follows from the eigenequation

$$
H_{F} \psi_{F}=E \psi_{F} .
$$

Inserting (A.7) into this equation, we obtain

$$
H \psi_{F}=\left(E+|\nabla F|^{2}-2 i A^{F}\right) \psi_{F} .
$$

Using this equation and the Schwarz inequality, we obtain

$$
\left|\left\langle\psi_{F}, H \psi_{F}\right\rangle\right| \leqq \frac{1}{4}\left\|\left.p \psi_{F}\right|^{2}+C\right\| \psi_{F} \|^{2} .
$$

As in Proposition A.1 one can show that

$$
-\left\langle\psi_{F}, V \psi_{F}\right\rangle \leqq \frac{1}{4}\left\|p \psi_{F}\right\|^{2}+C\|\| g\left\|^{1 / 2} \psi_{F}\right\|^{2} .
$$

This together with the equation

yields

$$
\left\|p \psi_{F}\right\|^{2}=\left\langle\psi_{F}, H \psi_{F}\right\rangle-\left\langle\psi_{F}, V \psi_{F}\right\rangle
$$

$$
\frac{1}{4}\left\|p \psi_{F}\right\| \leqq C\|\| g\left\|\left.\right|^{1 / 2} \psi_{F}\right\| \text {. }
$$

Thus $\psi_{F} \in D(p)$. Equation (A.9) shows that $\psi_{F} \in D(H)$. The case $n=2$ is proved in the same way.

Acknowledgements. It is a pleasure to thank E. Balslev, W. Hunziker. A. Jensen and A. Soffer for useful discussions and to thank $R$. Froese and $P$. Hislop for reading the manuscript and making useful remarks. The author is also grateful to the referee for helpful remarks and pointing our misprints as well as missing arguments (and, actually, supplying one argument). The hospitality at the Institute of Theoretical Physics, ETH Zürich, where most of this work was done, is gratefully acknowledged.

\section{References}

[A] Agmon, S.: Lower bounds for solutions of Schrödinger equations. J. d'Analyse Math. 23, $1-25(1970)$

[ABG] Amrein, W. O., Berthier, A. M., Georgescu. V.: $\mathrm{L}^{p}$-1nequalities for the Laplacian and unique continuation. Ann. Inst. Fourier, Grenoble 31, 153-168 (1981)

[AH] Avron, J. E., Herbst, I. W : Spectral and scattering theory of Schrödinger operators related to the Stark effect. Commun. Math. Phys. 52, 239-254 (1977)

[CFKS] Cycon, H. L., Froese, R. G., Kirsch, W., Simon, B.: Schrödinger Operators. Berlin, Heidelberg, New York: 1987

[FH] Froese, R., Herbst, I. W.: Exponential bounds and absence of positive eigenvalues for N-body Schrödinger operators. Commun. Math. Phys. 87, 429-447 (1982)

$\left[\mathrm{FH}(\mathrm{H}-\mathrm{O})^{2}\right]$ Froese, R., Herbst, I. W., Hoffmann-Ostenhof, M. T.: On the absence of positive eigenvalues for one-body Schrödinger operators. J. d'Analyse Math. 41, 272-284 (1982)

[HS] Herbst. I. W., Simon, B.: Dilation analyticity in constant electric field II. N-body problem, Borel summability. Commun. Math. Phys. 80, 181-216 (1981) 
[H] Hörmander, L:: Uniqueness theorems for second order elliptic differential equations Commun. Part. Diff. Eqns. 8, 21-64 (1983)

[Hu] Hunziker, W.: Notes on asymptotic perturbation theory for Schrödinger eigenvalue problems. Helv. Phys. Acta 61, 257-304 (1988)

[JK] Jerrison, D., Kenig, C. (with an Appendix by Stein, E. M.).: Unique continuation and absence of positive etgenvalues for Schrödinger operators. Ann. Math. 121, 463-494 (1985)

[L] Lavine, R.: Commutators and scattering theory I. Repulsive interactions. Commun. Math. Phys. 20, 301-323 (1971)

[RS] Reed, M., Simon, B.: Methods of modern mathematical physics II and IV, New York: Academic Press 1975 and 1978

[SchS] Schechter, M., Simon, B.: Unique continuation property for Schrödinger operators with unbounded potentials. J. Math. Anal. Appl. 77, 482-492 (1980)

[T] Titchmarsh, E. C.: Eigenfunction expansions associated with second order differential equations. Oxford University Press 1958

[Sig] Sigal, I. M.: Geometric theory of Stark resonances in multielectron systems. Comm. Math Phys. 119, 287-314 (1988)

Communicated by B. Simon

Recelved December 30, 1986; in revised form September 22, 1988 